\title{
Responsabilidad Social Universitaria. Una tarea urgente para las instituciones universitarias públicas de Medellín
}

\author{
University social responsibility. An urgent task for public \\ university institutions in Medellín
}

Recibido: 03/09/14 - Aprobado versión final: 20/10/14

Sandra Yaneth Rueda Villa*

\begin{abstract}
Resumen: en este artículo se analizan las prácticas y las políticas en materia de responsabilidad social que actualmente se desarrollan en las instituciones universitarias públicas de la ciudad de Medellín; de igual forma, se determina cómo está articulada la responsabilidad social universitaria - RSU - con la estructura organizativa de estas instituciones, y cómo ellas involucran a sus stakeholders en cada uno de los procesos relacionados con la RSU.
\end{abstract}

Palabras clave: ética, responsabilidad social, responsabilidad social universitaria, universidad, gestión organizacional.

\begin{abstract}
: this paper analyses social responsibility practices and policies under development among public university institutions in the city of Medellín. Also, the articulation of university social responsibility (USR) with organizational structure in the said institutions is established, as well as how they engage their stakeholders in all USR-related processes.
\end{abstract}

Keywords: ethics, social responsibility, university social responsibility, organizational management.

JEL: I23 - M14

\footnotetext{
* Ingeniera Administrativa, Magíster (C) en Administración de las Organizaciones y Coordinadora de Autoevaluación y Acreditación Institucional del Tecnológico de Antioquia, Medellín - Colombia. sandraruedavi@tdea.edu.co
} 


\section{Contexto Rued, s.}

\section{Responsabilité sociale des universités. Un impératif pour les institutions universitaires publiques de la ville de Medellin}

Résumé: dans cet article il est question d'analyser les pratiques et les politiques menés sous une perspective de responsabilité sociale à l'intérieur des institutions universitaires publiques de Medellin. Aussi nous nous penchons sur l'articulation entre la responsabilité sociale des universités (RSU) et la structure organisationnelle de ces institutions-là et sur la manière dont elles impliquent les stakeholders dans chacun des processus liés à la RSU.

Mots clé: éthique, responsabilité sociale, responsabilité sociale des universités, université, gestion organisationnelle.

\section{Introducción}

La universidad, como entorno social y cultural de masas, advierte en la sociedad del conocimiento de las relaciones entre los principales actores implicados en su devenir; pues la universidad ha de analizar los nuevos entornos y afrontar, con audacia, los retos de futuro desde una perspectiva de profundo compromiso con la función última que le es inherente: poner el conocimiento al servicio del ser humano, de su progreso ético, de su realización personal, desarrollo profesional y de la vinculación solidaria con los intereses generales. De aquí se deriva la importancia que está adquiriendo la metodología que se viene denominando el aprendizaje-servicio. La gestión interna de la universidad es una estrategia de responsabilidad social que se pone de manifiesto cuando la universidad es una organización en la que se constatan la democracia, la equidad (supresión de las segregaciones y corrección de los privilegios), transparencia (política y económica), haciendo de ella un modelo de desarrollo sostenible.

Tabla 1. Las distintas formas de comprender la universidad

\begin{tabular}{ll|}
\hline \hline Centrada en contenidos & Centrada en contenidos, actitudes y valores \\
\hline Formación de profesionales productivos. & $\begin{array}{l}\text { Formación de ciudadanos competentes en sus } \\
\text { funciones profesionales. }\end{array}$ \\
$\begin{array}{l}\text { Orientada a las necesidades del mercado } \\
\text { de trabajo. }\end{array}$ & $\begin{array}{l}\text { Orientada a anticiparse a las necesidades de la } \\
\text { sociedad en su conjunto. }\end{array}$ \\
$\begin{array}{l}\text { Uso social basado en el estatus, } \\
\text { enriquecimiento y reconocimiento } \\
\text { individual y en el crecimiento económico. }\end{array}$ & $\begin{array}{l}\text { Uso social basado en la contribución al bien } \\
\text { colectivo, a la construcción social y al desarrollo } \\
\text { humano. }\end{array}$ \\
\hline \hline
\end{tabular}

Fuente: propuesta de reporting de RSC en las universidades públicas. Aplicación al caso de la UNED (2008). 
La Responsabilidad Social Universitaria - RSU - supone rendir cuentas a la sociedad de los avances positivos y negativos respecto de los compromisos asumidos con sus grupos de interés y, en general, en materia de derechos humanos, medio ambiente, buen gobierno y compromiso social. La RSU consiste en hacer bien las cosas para todos, para ello se debe utilizar la transparencia en la gestión, ya que ésta atrae mayores aportes de financiación y patrocinios, y se crean una reputación y una buena imagen, mejoras de eficiencia en el uso de los recursos materiales (energéticos y otros), reducción de riesgos por conflictos con los grupos de interés (proveedores, empleados, clientes, entre otros) y la necesidad de generar valores inmateriales (excelencia docente e investigadora, valores humanos, etc..), y esa gestión de calidad llevará a mejores resultados en el futuro.

Se hace necesario considerar los aspectos relacionados con las acciones y las dimensiones de las universidades socialmente responsables, lo cual ha sido definido de la siguiente manera: "Las acciones llevadas a cabo por la universidad se identifican a través de los efectos o impactos que generan en la sociedad". Las mismas se agrupan en tres ámbitos de actuación, y en relación con ellos indicamos lo siguiente (UMA, 2007):

\section{Medio ambiente}

Comprende todas aquellas acciones realizadas por la universidad que han tenido o han podido tener un impacto sobre los sistemas naturales vivos e inertes, incluidos los ecosistemas, el suelo, el aire y el agua.

\section{Social}

Incorpora la totalidad de las acciones llevadas a cabo por la universidad que han tenido o han podido tener un impacto sobre las condiciones sociales de sus grupos de interés, tales como alumnos, personal docente e investigador, personal de administración y servicios, equipo de gobierno, proveedores, egresados, empresarios, representantes sociales, representantes institucionales, medios de comunicación, entre otros.

\section{Económico}

Abarca todas las acciones efectuadas por la universidad que han tenido, o han podido tener un impacto sobre las condiciones económicas de sus grupos de interés y de los sistemas económicos autonómico, local, nacional y mundial.

Existen diversas acciones para que la universidad demuestre su compromiso con la sociedad, aunque cada universidad podrá optar por enfoques distintos según sus prioridades, las de sus grupos de interés y las del entorno en el que desarrolla sus actividades. En la Tabla 2 podrán analizarse algunas de las acciones que pueden llevar a cabo aquellas universidades que son socialmente responsables y contribuyen al desarrollo sostenible. 


\section{Contexto Rueda, $S$}

Tabla 2. Acciones en materia de responsabilidad social de las universidades

\begin{tabular}{|c|c|c|c|}
\hline $\begin{array}{l}\text { Dimensiones } \\
\text { y ámbitos }\end{array}$ & $\begin{array}{l}\text { Enseñanza- } \\
\text { aprendizaje } \\
\text { I+D+i }\end{array}$ & Gestión & Relaciones con la sociedad \\
\hline $\begin{array}{c}\text { Medio } \\
\text { ambiente }\end{array}$ & $\begin{array}{l}\text { Crear conocimiento } \\
\text { transdisciplinar } \\
\text { y modelos para } \\
\text { el desarrollo } \\
\text { sostenible. } \\
\text { Generar tecnologías } \\
\text { y conocimientos } \\
\text { que se puedan } \\
\text { aplicar al medio } \\
\text { ambiente. }\end{array}$ & $\begin{array}{l}\text { Reducir el uso } \\
\text { y consumo de } \\
\text { recursos naturales. } \\
\text { Fomentar medios } \\
\text { de transporte que } \\
\text { sean sostenibles. } \\
\text { Promover el } \\
\text { reciclaje. } \\
\text { Reducir los } \\
\text { residuos. }\end{array}$ & $\begin{array}{l}\text { Cuidar y conservar las zonas } \\
\text { ecológicas en las universidades. } \\
\text { Promover proyectos de } \\
\text { protección del medio ambiente. } \\
\text { Informar sobre la sostenibilidad. }\end{array}$ \\
\hline Social & $\begin{array}{l}\text { Formar en valores. } \\
\text { Investigar en } \\
\text { materia de } \\
\text { responsabilidad } \\
\text { social. } \\
\text { Crear modelos de } \\
\text { actuación y gestión. }\end{array}$ & $\begin{array}{l}\text { Gestionar los } \\
\text { riesgos laborales. } \\
\text { Promover la } \\
\text { igualdad de } \\
\text { oportunidades. } \\
\text { Invertir en la } \\
\text { formación de los } \\
\text { profesionales. }\end{array}$ & $\begin{array}{l}\text { Cooperar con la sociedad. } \\
\text { Generar empleo. } \\
\text { Promover el debate de la } \\
\text { responsabilidad social en la } \\
\text { sociedad para concientizarla. }\end{array}$ \\
\hline Económico & $\begin{array}{l}\text { Implantar como } \\
\text { tema transversal } \\
\text { en la formación, } \\
\text { el estudio de } \\
\text { temas relativos a } \\
\text { la responsabilidad } \\
\text { social. }\end{array}$ & $\begin{array}{l}\text { Mejorar la } \\
\text { eficiencia en el } \\
\text { uso de edificios y } \\
\text { tecnología. } \\
\text { Establecer códigos } \\
\text { de conducta. }\end{array}$ & $\begin{array}{l}\text { Fomentar inversiones basadas } \\
\text { en criterios éticos y socialmente } \\
\text { responsables. }\end{array}$ \\
\hline
\end{tabular}

Fuente: adaptado a partir de Latorre (2006).

Ahora bien, las acciones de la universidad son muy numerosas y variadas, siendo necesario establecer dentro de cada ámbito de actuación dimensiones en materia de responsabilidad social para poder clasificarlas. Dichas dimensiones son las siguientes (UMA, 2007):

- Enseñanza-aprendizaje e investigación, desarrollo tecnológico e innovación $(\mathrm{I}+\mathrm{D}+\mathrm{i})$.

- Gestión.

- Relaciones con la sociedad. 
En relación con lo expuesto destacamos, por la relevancia que presenta actualmente, que en la dimensión relaciones con la sociedad se incluyen las actuaciones relacionadas con la transferencia de conocimiento y la extensión cultural. Las primeras son fundamentales para incrementar la innovación del sistema productivo y los servicios, mientras las segundas permiten la proyección de las propias universidades. Por consiguiente, tanto las actividades de transferencia como las de extensión son de tal relevancia que podrían, por sí mismas, constituir dos nuevas dimensiones por considerar en cada uno de los ámbitos de la responsabilidad social; además de las acciones que se pueden llevar a cabo según los ámbitos y las dimensiones que hemos analizado, el principal objetivo de la universidad, al igual que el de cualquier otra organización, se concreta en el desarrollo sostenible y en la generación de valor. (Jiménez, 2008)

Ante las vertiginosas transformaciones de un mundo globalizado, el papel de las universidades es fundamental para la formación de capacidades y valores que brinden respuestas a las necesidades y exigencias de la sociedad, y propicien una cultura de responsabilidad social en el ejercicio de la profesión. En la actualidad ha cobrado una amplia difusión el uso de índices integrales para evaluar determinados aspectos de la gestión empresarial. Se propuso el diseño de un índice integral para evaluar la Responsabilidad Social Universitaria, a través de los procesos sustantivos de docencia, investigación, gestión, vinculación con la colectividad y dos perspectivas esenciales para la gestión de la responsabilidad social: ambiental y comunicacional.

Resulta evidente, en atención a lo planteado, la necesidad de modificar paradigmas dentro de las universidades, que abran paso a un nuevo modelo de gestión universitaria, para lo cual es imprescindible el impulso de procesos innovadores dentro de las organizaciones, destinados a lograr cambios sustantivos en los esquemas tradicionales y en la forma de relacionarse las universidades con el entorno socioproductivo. (Ramos, 2010)

\section{Metodología}

En primer lugar, es importante mencionar el tipo de estudio que se va a realizar en la investigación, que para efectos del presente artículo se ha establecido como una investigación de tipo descriptivo. El diseño con el cual se elabora este proceso de es no experimental. De esta manera, la investigación se centra en analizar cuál es el nivel o el estado de diversas variables en un momento dado o bien, cuál es la relación entre un conjunto de variables en un punto en el tiempo.

En este caso el diseño apropiado (bajo un enfoque no experimental) es el transversal o transicional. Este diseño permite la toma única de datos que se realiza por medio de un instrumento de tipo encuesta, basado en factores clave y de gran relevancia en el estudio de la implementación 


\section{Contexto Rued,s}

de responsabilidad social empresarial en las instituciones universitarias públicas de la ciudad de Medellín. El diseño requiere, en primer lugar, la selección de una muestra intencionada para la aplicación de los instrumentos, con una población con características y conocimientos específicos, que conozca el tema, que permita hallar una información representativa y con un margen de error mínimo. Por ejemplo, los Comités Paritarios de Salud Ocupacional - COPASO -, son comités compuestos por varios empleados que estarían en capacidad de responder la encuesta, con el objetivo de servir de instrumento exploratorio para ayudar a identificar variables, conceptos básicos, herramientas, prácticas y políticas en materia de responsabilidad social en instituciones públicas de educación superior de Medellín. Las preguntas diseñadas permiten medir las variables propuestas en esta primera investigación, para realizar un diagnóstico inicial sobre el conocimiento de la responsabilidad social en el grupo de estudio.

\section{Resultados}

La responsabilidad social es un tema que día a día toma fuerza en las organizaciones privadas y públicas, y genera impacto a nivel social, económico, ambiental, cultural y comunitario, al desarrollar un valor agregado que le permite competir con altura en el exigente mundo globalizado. Es un compromiso de las empresas contribuir al desarrollo económico sostenible, cumplir con las expectativas que generan en la sociedad y con la rendición de cuentas frente al impacto que deberían tener como organización frente a sus públicos, a la sociedad civil y al Estado.

En tal sentido, Sánchez Arza plantea que,

En las instituciones de educación superior se busca identificar su rol en el contexto de la responsabilidad social empresarial; es menester conocer los orígenes de este concepto y sus alcances en el marco de la sociedad actual, por lo que se considera importante mencionar algunos conceptos que los autores han empleado para referirse al tema. (2011)

Es importante entonces que para el análisis de los resultados de investigación obtenidos en este proceso, se parta por el reconocimiento de la población y la muestra que se trabajó, de tal manera que la exposición de los resultados sea clara para cumplir con el propósito principal planteado en este artículo. La población con la cual se trabajó fue la del Tecnológico de Antioquia Institución Universitaria, el Politécnico Colombiano Jaime Isaza Cadavid, el Instituto Tecnológico Metropolitano, el Instituto Tecnológico Pascual Bravo y el Colegio Mayor de Antioquia. La encuesta se realizó a 28 personas que trabajan en las instituciones universitarias públicas de la ciudad de Medellín, las cuales tenían diferentes roles como docentes, personal administrativo, personal del área contable y mensajeros. 
A continuación se presentan los resultados obtenidos en cada una de las consultas realizadas a los informantes clave del proceso.

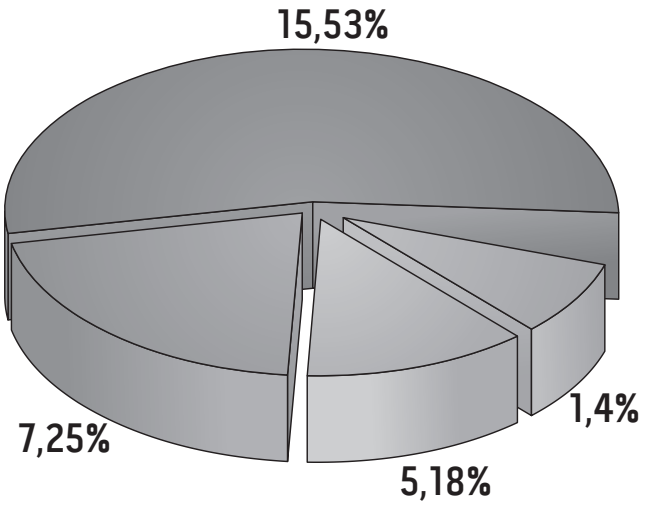

a. La RS es voluntaria, es reconocer y aceptar los compromisos que tenemos con nuestra sociedad.

b. La RS es el compromiso que asume una organización para contribuir al desarrollo económico de sus empleados

c. La RS es el conjunto de prácticas empresariales abiertas y transparentes basadas en valores éticos y en el respeto a nivel social, y con el medio ambiente

d. La RS es una interacción voluntaria por parte de las empresas en temas sociales y ambientales, y sus operaciones comerciales

Figura 1. Imaginarios de la Responsabilidad Social

Fuente: construcción propia con base en las encuestas realizadas en las instituciones universitarias públicas de la ciudad de Medellín.

El 53\% de los encuestados reconocen la responsabilidad social como un conjunto de prácticas empresariales, abiertas y transparentes, basadas en valores éticos y en el respeto social y al medio ambiente. Luego, el $25 \%$ consideran que la RS es el compromiso que asume una organización para contribuir al desarrollo económico de sus empleados; y solamente un 3,5\% la refirió como una interacción voluntaria por parte de las empresas, en temas sociales y ambientales y sus operaciones comerciales. De los encuestados, el porcentaje más alto seleccionó la respuesta $C$; esto implica que la mayoría sienten que la responsabilidad social, más que un compromiso, un reconocimiento o una interacción, es una práctica que se debe evidenciar en el actuar, apoyando un tema que se debe reforzar, como es el de los valores éticos y el respeto, no solo por el entorno social, sino también por el medio ambiente. 


\section{Contexto Ruedas.}

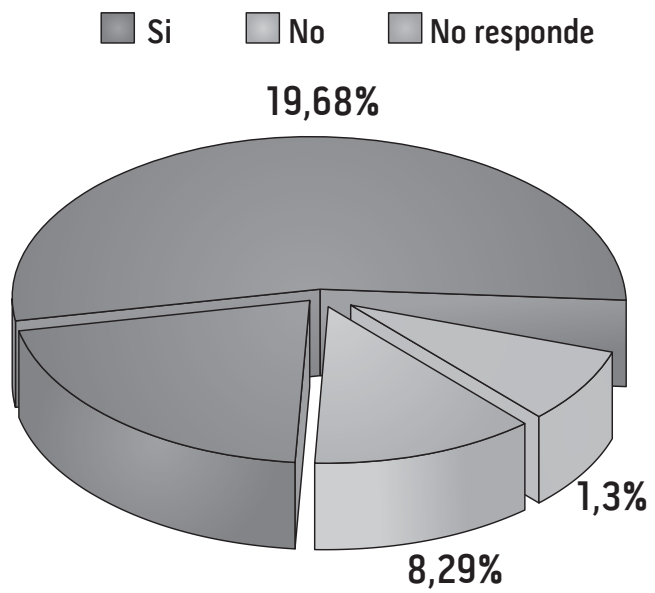

Figura 2. RSU Institucional

Fuente: construcción propia con base en las encuestas realizadas en las instituciones universitarias públicas de la ciudad de Medellín.

Aunque el 68\% de los encuestados reconocen que se tiene en cuenta la responsabilidad social en las instituciones universitarias, existe aún un alto porcentaje (29\%) de los encuestados que manifiestan no conocer la responsabilidad social en la institución, lo que permite evidenciar que se hace necesario crear estrategias de difusión y de las diferentes actividades que se realizan con relación al tema.

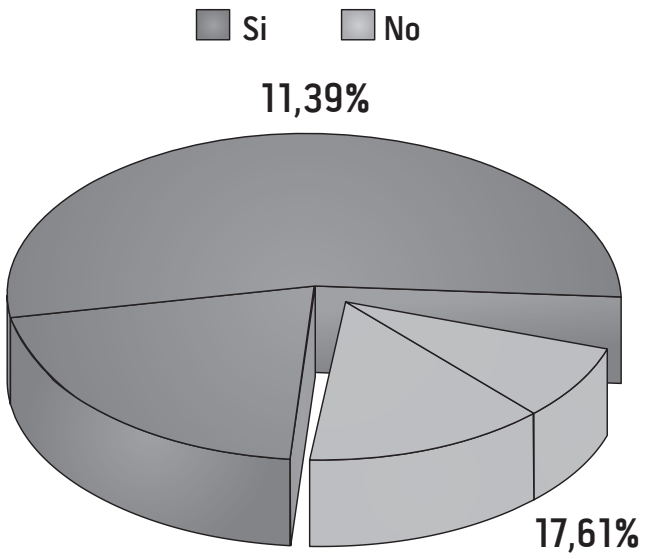

Figura 3. Políticas y prácticas de RSU

Fuente: construcción propia con base en las encuestas realizadas en las instituciones universitarias públicas de la ciudad de Medellín. 
El $61 \%$ de las personas entrevistadas manifiestan que en las instituciones universitarias, dentro de sus políticas y prácticas, algunas incluyen el tema de responsabilidad social, lo cual permite evidenciar que, aunque dentro de las políticas estatales no existe una obligación expresa de incluir dentro del plan de desarrollo de las instituciones como premisa la responsabilidad social, existe un alto porcentaje de las mismas que asumen el compromiso con la comunidad que impactan. También es claro, por lo que expresa la gráfica, que aún es muy alto el porcentaje (39\%) de instituciones universitarias públicas que no incluyen dentro de sus políticas y prácticas la responsabilidad social como elemento fundamental para la sostenibilidad $\mathrm{y}$ visibilidad en el mercado, es importante que desde el Estado se generen políticas y estrategias que garanticen la inclusión de la responsabilidad social como premisa que oriente su quehacer.

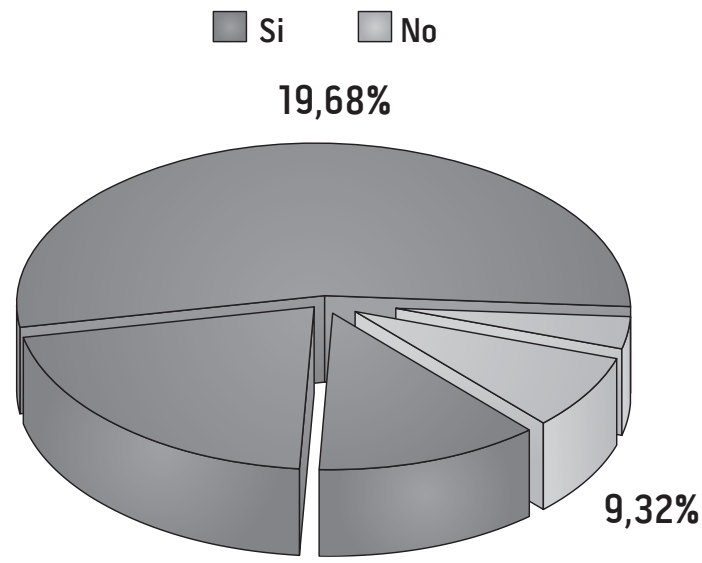

Figura 4. Beneficios de la RSU

Fuente: construcción propia con base en las encuestas realizadas en las instituciones universitarias públicas de la ciudad de Medellín.

El $68 \%$ de la población encuestada manifiesta que se ha beneficiado con los programas que implementa la institución para contribuir con el desarrollo social, beneficio expresado en garantías de bienestar integral para la comunidad educativa. Aún existe un alto porcentaje (32\%) que falta por impactar, si se tiene en cuenta la importancia de que las instituciones universitarias realicen un diagnóstico de las necesidades de la población académica. 


\section{Contexto ruvedas}

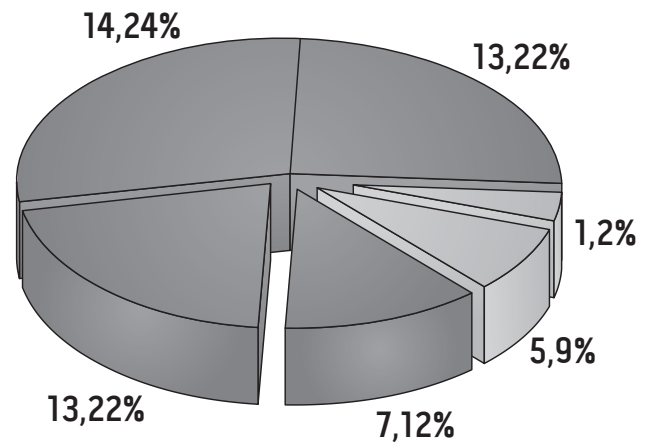

$\square 1 \square 2 \square 3 \quad \square 4 \quad \square 5 \quad \square 6$
1. Ética, valores y principios de los negocios

2. Derechos humanos, trabajo y empleo

3. Gobernabilidad corporativa

4. Impacto sobre el medio ambiente

5. Relaciones con proveedores

6. Filantropía e inversión social

7. Transparencia y rendición de cuentas

Figura 5. Factores de RSU visibilizados en las instituciones universitarias públicas

Fuente: construcción propia con base en las encuestas realizadas en las instituciones universitarias públicas de la ciudad de Medellín.

Los encuestados dan un alto grado de relevancia, con un porcentaje del $24 \%$, al compromiso que asumen las universidades con la preservación del medio ambiente, como componente fundamental de la responsabilidad social.

De otro lado, un $44 \%$ de los encuestados relacionan los derechos humanos, el trabajo, el empleo, la ética (valores) y los principios de los negocios, que se incluyen actualmente como factores relevantes en la institución universitaria, lo cual indica la integralidad de elementos que hacen parte de las diferentes estrategias por desarrollar en los programas de responsabilidad social.

Cabe destacar que el $21 \%$ de los encuestados le dan importancia a la transparencia en el manejo administrativo de los recursos institucionales, que pone en evidencia un factor importante como componente de la responsabilidad social. 


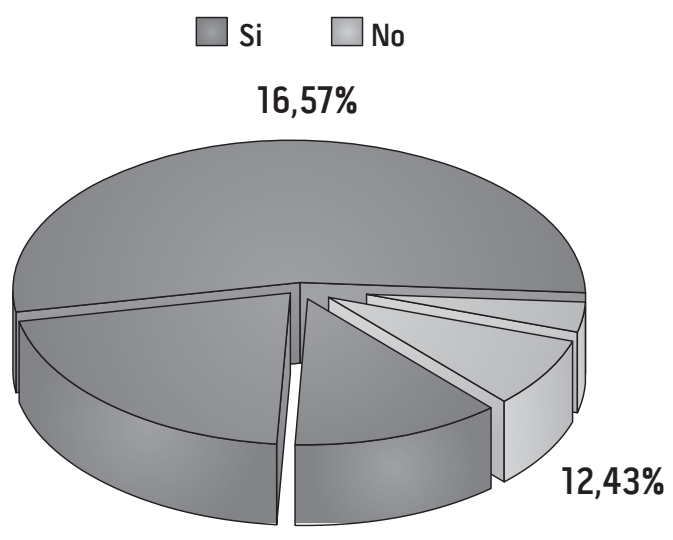

Figura 6. Investigación y RSU

Fuente: construcción propia con base en las encuestas realizadas en las instituciones universitarias públicas de la ciudad de Medellín.

El 57\% de las personas encuestadas manifiestan que las instituciones universitarias han incluido en los proyectos de investigación desarrollados el tema de la responsabilidad social, como respuesta al compromiso que asumen a través de sus procesos misionales: la docencia, la investigación y la extensión. Sin embargo, existe un alto porcentaje (43\%) de instituciones que aún no incluyen el compromiso social como un lineamiento en sus proyectos de investigación.

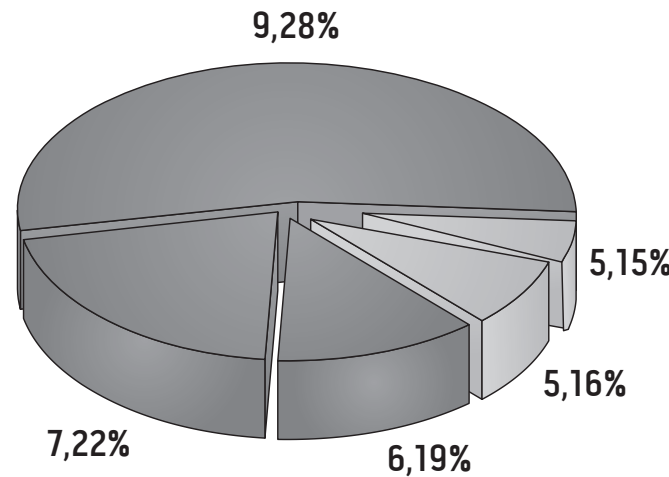

$6,19 \%$ a. A nivel social

b. A nivel de los empleados

c. A nivel de la comunidad

d. A nivel ambiental

e. A nivel de transparencia y claridad administrativa

Figura 7. Impacto investigativo y RSU

Fuente: construcción propia con base en las encuestas realizadas en las instituciones universitarias públicas de la ciudad de Medellín. 


\section{Contexto ruedas.}

El 65\% de los encuestados manifiestan que el mayor impacto de la responsabilidad social de los diferentes proyectos de investigación se orienta a los ámbitos social y comunitario; lo que demuestra que la percepción de la responsabilidad social está encausada al compromiso con el ser humano.

De otro lado, se evidencia la asociación que existe entre el compromiso social de las instituciones y la preservación del medio ambiente con los programas de desarrollo sostenible, emanado de algunas instituciones, como aspecto preponderante en sus proyectos socioambientales, con un porcentaje del $19 \%$ con el tema ambiental.

\section{Discusión de los resultados más apremiantes}

Los cambios socioeconómicos acontecidos en Colombia durante los últimos años han alterado drásticamente la dinámica de las organizaciones, han aumentado la competencia y la competitividad, exigiéndoles a las mismas un cambio estratégico enfocado en el desarrollo de la innovación, pero además se ha involucrado considerablemente la responsabilidad social como un elemento fundamental para la sostenibilidad y la visibilidad en el mercado, en aras de lograr un cambio social y una transformación en el medio ambiente y en el desarrollo sostenible.

Teniendo en cuenta que las universidades son un elemento clave para mejorar la productividad y la competitividad de la economía, y más en el contexto de la globalización actual, y que estas se encuentran en transición por los cambios en el mundo productivo, la evolución tecnológica y la sociedad de la información, se hace necesario que trabajen arduamente hacia el ideal de una sociedad del conocimiento, y en la formación de profesionales íntegros, éticos y preparados para enfrentar estos cambios. Para ello se requiere, por parte de las instituciones de educación superior - IES -, no solo una responsabilidad académica sino también una responsabilidad social.

Por tal razón, las IES se han preocupado por generar esfuerzos a través de estrategias, políticas y acciones orientadas a la responsabilidad social universitaria, lo que ha permitido redireccionar sus objetivos y su misión hacia unas prácticas responsables, con el fin de garantizar la calidad de los sistemas de educación y abarcar de forma transversal las funciones propias de las IES, como lo son la docencia, la investigación, la extensión y la gestión.

La incorporación e implementación de la responsabilidad social en las instituciones de educación superior es esencial, ya que son entes generadores de nuevos conocimientos y de profesionales que deben ser capaces de desarrollar mecanismos orientados a la transformación social, política y económica que demanda la sociedad. Desde este enfoque, es sumamente importante que las IES empiecen a darle mayor fuerza a este tema a través de convenios, alianzas estratégicas, redes, grupos de investigación y mecanismos formales de participación social y ciudadana. En tal sentido, la 
universidad debe ser un medio fundamental para que nuestra ciudad, nuestra región y nuestro país se transformen productiva y socialmente.

La investigación tiene un papel muy importante con relación a la responsabilidad social, ya que a través de los proyectos o propuestas que se generen en las instituciones se pueden incorporar estrategias eficaces para lograr un continuo desarrollo de este concepto, orientadas hacia una visión integradora de las dimensiones sociales, económicas y ambientales.

Los resultados obtenidos en el desarrollo de esta investigación muestran la importancia que empieza a cobrar para las instituciones universitarias públicas de la ciudad de Medellín la inclusión del concepto de responsabilidad social, porque proporciona herramientas con impacto positivo al servicio de una educación integral, que forma profesionales con valores éticos y sociales y con las competencias necesarias para afrontar los retos personales y fortalecer el conocimiento científico y tecnológico. Sin embargo, se requiere la aplicación de los elementos descritos en el análisis de la información, que se presentan en este escrito, para así garantizar la pertinencia en un entorno social, natural y humano.

\section{Conclusiones}

El recurso humano encuestado visualiza la responsabilidad social como un compromiso a nivel empresarial, es decir, debe partir de la alta gerencia para con los demás, pero no tienen la conciencia de que la responsabilidad social es un asunto de todos, involucra a cada uno de los miembros de la comunidad académica, y lo más importante, es un asunto en doble dirección: de la empresa a la sociedad y de la sociedad a la empresa.

Entre los resultados obtenidos a través de la aplicación del instrumento se observa que existe una percepción de que la responsabilidad social está orientada fundamentalmente a temas ambientales, sociales y éticos, pero existe la convicción de que no involucra a los proveedores, cuando es un asunto de interés y competencia de toda la sociedad.

El estudio realizado pone en evidencia que el concepto de responsabilidad social es conocido en el medio, pero no se tiene la suficiente claridad para identificar programas y políticas que tengan que ver con el tema.

Según los resultados, se puede concluir que un alto porcentaje de las personas encuestadas tienen que ver con proyectos de investigación en las instituciones educativas, y en éstos se ha tenido presente el concepto de la responsabilidad social como compromiso institucional con la comunidad y con la sociedad en general.

Los entrevistados perciben que, en el ámbito investigativo, las instituciones apuestan por el impacto social más que por otros temas, en relación con el concepto de la responsabilidad social. 


\section{Contexto Rueda, $S$.}

Aunque las personas encuestadas reconocen que las instituciones de educación superior incluyen en sus programas actividades encaminadas al reconocimiento y la inclusión del concepto de responsabilidad social, se percibe que algunas personas no poseen la claridad suficiente en torno al concepto.

En general, según los resultados arrojados por las encuestas a personas relacionadas con las diferentes instituciones públicas de educación superior de Medellín, se evidencia que se trabaja el concepto de responsabilidad social y que se ha generado un impacto positivo sobre la población.

Aunque es manifiesto el conocimiento de que las instituciones de educación superior tienen programas encaminados hacia la responsabilidad social, aún hay personas que ven que no han sido beneficiados.

En general, según los resultados arrojados por las encuestas a personal relacionado con las diferentes instituciones públicas de educación superior de Medellín, se detecta que se está trabajando los temas de responsabilidad social, y que se ha generado un impacto positivo sobre la población; sin embargo, aún se evidencia la falta de capacitación y comunicación sobre el tema.

\section{Recomendaciones para el mejoramiento de la RSU}

Entre las recomendaciones que realizaron los encuestados, se encuentran:

- Todas las personas que trabajan en las instituciones educativas deben tomar conciencia de la responsabilidad social personal, y orientada hacia la comunidad.

- La tecnología es una herramienta que debe hacer parte de las políticas y programas de responsabilidad social.

- En las instituciones educativas se deben generar espacios de formación en bioética social, derechos humanos y responsabilidad social.

- Todo proceso de investigación debe estar orientado hacia alguno de los componentes de la responsabilidad social.

- En las instituciones educativas las campañas deben estar orientadas en conjunto: Docentes, estudiantes y equipos administrativos.

- Bienestar institucional debería generar herramientas de comunicación para la divulgación y socialización de los proyectos.

- Se deben generar espacios de capacitación sobre los temas relacionados con la responsabilidad social.

En general, según los resultados arrojados por las encuestas aplicadas al personal relacionado con las diferentes instituciones universitarias públicas de Medellín, se detecta que se está trabajando en los temas de responsabilidad social, y que se ha generado un impacto positivo sobre la población; se observa un buen conocimiento y aplicación del tema, buen manejo en los 
procesos de comunicación y socialización, por medio de las prácticas y acciones que se están implementando en dichas instituciones educativas.

Con relación al presente estudio se realizan unas recomendaciones orientadas a la implementación de estrategias para la capacitación y concientización de las comunidades en torno a la necesidad, obligatoriedad y compromisos de la responsabilidad social en las entidades, y fundamentalmente en las instituciones de educación superior.

En las instituciones universitarias públicas se debe fortalecer la inclusión de políticas que involucren a toda la comunidad académica, orientadas a la responsabilidad social y a la implementación de estrategias que, en primer lugar, inviten a la participación y construcción de dichas políticas, y que, finalmente, den origen a la sensibilización y concientización sobre la importancia y el compromiso que tiene, a nivel mundial, la educación con los procesos sociales.

Las IES públicas deben ofertar programas que permitan dar cobertura a la educación superior, en las diferentes regiones de cada localidad, y sobre todo apertura de programas que respondan a las necesidades de la comunidad o contexto, con currículos pertinentes para la realidad que exige el mundo de hoy y con las condiciones generales suficientes para impartir una educación con calidad. De igual forma, hacer los ajustes necesarios a los programas ya existentes, y abrir nuevos cuyo sello principal sea la inmersión educativa con visión social.

Se hace necesario examinar y reconstruir la normativa universitaria en relación con las nuevas realidades socioeconómicas del país, a través del diseño de diferentes estrategias que propendan por una identidad universitaria y un compromiso social, que propongan un mecanismo de integración con el entorno de la universidad.

Es importante constituir una relación permanente con otras universidades y con el sector productivo a nivel regional, departamental, nacional e internacional, que permita establecer alianzas estratégicas en función del desarrollo del país, y definir políticas de formación permanente y de reconocimiento de méritos dirigidas a los diferentes grupos que despierten niveles de competitividad, y de responsabilidad social, de acuerdo con las necesidades reales de la comunidad. Finalmente, es recomendable que las instituciones universitarias públicas aumenten la financiación y los estímulos con relación a los proyectos de investigación, con pertinencia social e impacto comunitario.

\section{Referencias bibliográficas}

Barreto, P. (2007). Responsabilidad social universitaria. Recuperado de http://www.newsperuvian.com/Portal/index.php?option=com_conte nt\&task=view $\&$ id $=24 \&$ Itemid $=100$ 


\section{Contexto Ruedas.}

Castañeda, G., Ruiz, M., Viloria, O., Castañeda, R. \& Quevedo Y. (2007). El rol de las universidades en el contexto de la responsabilidad social empresarial. Revista Negotium, 3(8). Recuperado de http://www. revistanegotium.org.ve/pdf/8/Art4.pdf

Jiménez de Lajara, M. (2008). ¿Cómo medir la percepción de la responsabilidad social en los diversos estamentos de la universidad?: una experiencia concreta. Educación Superior y Sociedad, 2, 139-162.

La Torre, F. (2006). Universidades responsables. La responsabilidad social corporativa en la Universidad de Zaragoza. Toledo, Ponencia presentada en el IX Congreso Estatal del Voluntariado.

Pelekais, C., Ferrer, J., Cruz, A. \& Romero, D. (2007). Responsabilidad social: compromiso de las organizaciones. Maracaibo: Ediciones Astro Data, S.A.

Ramos, C. (2010). Hacia una cultura de responsabilidad social universitaria. Publicaciones URBE, 7(2), 97-113. Recuperado de http://www. publicaciones.urbe.edu/index.php/cicag/article/viewArticle/364/867

Sánchez, C. (2011). El rol de las universidades en el contexto de la responsabilidad social. II Congreso Internacional IGLU. Recuperado de https://repositorio.ufsc.br/bitstream/handle/123456789/32865/8.13. pdf? sequence $=1$

UMA. (2007). Presentación al Claustro de la Memoria de Responsabilidad Social de la Universidad de Málaga 2006-2007. (Documento sin publicar). Málaga, Vicerrectorado de Coordinación Universitaria. Secretariado de Calidad y Planificación Estratégica. Universidad de Málaga.

Vallaeys, F. (2008). Responsabilidad social universitaria: una nueva filosofía de gestión ética e inteligente para las universidades. Educación Superior y Sociedad, 2, 191-220.

\section{Para citar este artículo:}

Rueda, S. (2014). Responsabilidad Social Universitaria. Una tarea urgente para las instituciones universitarias públicas de Medellín. En-Contexto, 2, 199-214. 